\title{
THE IMMUNOCYTOCHEMICAL LOCALIZATION OF TYROSINE HYDROXYLASE WITHIN RAT SYMPATHETIC NEURONS THAT RELEASE ACETYLCHOLINE IN CULTURE ${ }^{1}$
}

\author{
D. HIGGINS, ${ }^{2}$ L. IACOVITTI, T. H. JOH, ${ }^{*}$ AND H. BURTON \\ Departments of Anatomy and Neurobiology and Physiology.Biophysics, Washington University School of Medicine, St. Louis, Missouri \\ 63110, and *Cornell University Medical College, New York, New York 10021
}

\begin{abstract}
In vitro populations of neurons dissociated from the superior cervical ganglion of the rat embryo can acquire many of the properties of cholinergic neurons and yet retain some of the characteristics of adrenergic neurons. To determine whether one neuron can stably express properties of two transmitter systems, identified neurons were characterized by both immunocytochemical and electrophysiological methods. It was found that more than $90 \%$ of the neurons that formed functional cholinergic synapses had tyrosine hydroxylase within their cytoplasm. Furthermore, this mixed phenotypic expression persisted for more than 1 month. It is concluded that, under certain conditions in vitro, most neurons from the superior cervical ganglion have at least the potential for dual function.
\end{abstract}

Populations of the principal neurons from the superior cervical ganglion (SCGN) of the rat embryo can, under certain conditions in vitro, gradually acquire many of the characteristics of cholinergic neurons (reviewed by Patterson, 1978; Burton and Bunge, 1980). Thus, in dissociated cell cultures, the SCGN form nicotinic cholinergic synapses (O'Lague et al., 1974, 1978b; Ko et al., 1976), choline acetyltransferase activity increases more than 1000-fold (Patterson and Chun, 1977; Johnson et al., 1980 ), and the synaptic terminals come to contain synaptic vesicles with clear cores (Johnson et al., 1980). In these same cultures, the sympathetic neurons continue to express many of the characteristics expected of adrenergic neurons, such as the capacity to transport (Patterson et al., 1975; Reichardt and Patterson, 1977; Wakshull et al., 1978) and release norepinephrine (Burton and Bunge, 1975; Patterson et al., 1975). Two different cellular mechanisms are currently being considered as explanations for these changes in the properties of the population of dissociated SCGN (reviewed by Patterson, 1978; Burton and Bunge, 1980). It is possible that individual neurons can stably express only one transmitter system, either adrenergic or cholinergic; the gradual acquisition of cholinergic traits in the culture could be accomplished by individual neurons forsaking the adre-

\footnotetext{
'We are grateful to $R$. P. Bunge for reading and discussing this manuscript and to S. Mantia for secretarial aid. This work was supported by National Institutes of Health Grants NS 09808, MH 24285, NB 11888, and NB 09923.

${ }^{2}$ To whom correspondence should be addressed at Department of Anatomy and Neurobiology, Washington University School of Medicine, 660 South Euclid Avenue, St. Louis, MO 63110.
}

nergic phenotype and adopting a cholinergic phenotype. Alternatively, the changes in the properties of the population may reflect alterations within single neurons. Thus, individual cells may retain some or all of the traits of an adrenergic neuron and, in addition, acquire characteristics of a cholinergic neuron.

Single sympathetic neurons capable of synthesizing (Reichardt and Patterson, 1977) or releasing (Furshpan et al., 1976) both norepinephrine and acetylcholine have been observed in vitro. Since the neurons that synthesized both transmitters constituted only a small minority of the population, there was speculation that these rare dual function neurons might be in the process of changing from an adrenergic to a cholinergic phenotype (Reichardt and Patterson, 1977). This matter was not resolved, however, because there was the possibility that the biochemical methods were unable to detect a small amount of one of the transmitters in a cell that was actually bifunctional (Patterson, 1978).

Observations have been made supporting the hypothesis that properties of both transmitter systems may persist in the same cell. Thus, most of the neurons that synthesized acetylcholine in these kinds of cultures retained the transport mechanism for catecholamines (Reichardt and Patterson, 1977; Wakshull et al., 1978). Furthermore, within cultures containing predominantly cholinergic neurons, there were at least a few synaptic vesicles that reacted with a cytochemical stain for catecholamines (Johnson et al., 1980) after exposure to norepinephrine. Finally, in cultures in which there were high levels of choline acetyltransferase activity, greater than $95 \%$ of the neurons contained the specific catecholaminesynthesizing enzyme, tyrosine hydroxylase (TH); the 
amount and activities of TH and dopamine $\beta$-hydroxylase also increased in parallel with the increased level of choline acetyltransferase activity in these same cultures (Iacovitti et al., 1980).

In this paper, we report the results of experiments in which single identified neurons were studied with both immunocytochemical and electrophysiological techniques. These experiments provide evidence that individual sympathetic neurons release acetylcholine in culture and yet retain the rate-limiting enzyme for the synthesis of norepinephrine.

\section{Materials and Methods}

Neurons dissociated from the superior cervical ganglion of rat embryos (19 to $21 \mathrm{~d}$ ) were maintained in vitro in the absence of non-neuronal cells for up to 8 weeks by procedures that have been previously described (Wakshull et al., 1978). Neurons were plated at low density $\left(<2\right.$ neurons $\left./ \mathrm{mm}^{2}\right)$ on an air-dried collagen substratum; neuronal densities below 1000 neurons per dish facilitated the detection of cholinergic synapses (Ko et al., 1976; D. Higgins, unpublished observations). After the 3rd day in vitro, the cells were maintained in a nutrient medium containing 65\% Eagle's minimal essential medium with added glutamine (1.4 mmol/liter) and glucose $(33 \mathrm{mmol} /$ liter), $25 \%$ human placental serum, $10 \%$ chick embryo extract, and nerve growth factor ( 25 units $/ \mathrm{ml}$ prepared by the method of Bocchini and Angeletti (1969) and assayed by the method described by Varon et al. (1972)); before this time, the cultures were maintained in a medium (Medium B in Table I of Wakshull et al., 1978) that contained an antimitotic agent.

The experimental protocol consisted of obtaining simultaneous electrophysiological recordings from pairs of neurons that were identified from photomicrographs. At the end of the recording session, the cultures were promptly processed for the immunocytochemical location of $\mathrm{TH}$, and these neurons were relocated. The stain- ing properties of the physiologically studied neurons were then compared to the immunocytochemical reactions in adjacent, non-impaled neurons and to the reactions of neurons in control cultures (see below).

The electrophysiological methods were similar to those previously described (Ko et al., 1976); however, several modifications were made that, together with the use of low density cultures, have allowed us to detect a higher incidence of synaptic interactions than in previous reports. First, recordings were obtained with hyperfine micropipettes (l-mm Omega Dot glass pulled to resistances of 125 to 175 megohms on a Brown-Flaming Micropipette Puller). Second, the perfusion system was improved to allow rapid (less than $3 \mathrm{~min}$ ) changes of media so that a larger number of synaptic connections could be tested pharmacologically. The cultures were also maintained in a more excitable condition by perfusion with a balanced salt solution $(120 \mathrm{~mm} \mathrm{NaCl}, 12 \mathrm{~mm}$ $\mathrm{NaOH}, 30 \mathrm{~mm}$ HEPES (4-(2-hydroxymethyl)-1-piperazineethanesulfonic acid), $1 \mathrm{~mm} \mathrm{NaH} \mathrm{PO}_{4}, 2 \mathrm{~mm} \mathrm{\textrm {CaCl } _ { 2 } , 5}$ $\mathrm{mm} \mathrm{KCl}, 11 \mathrm{~mm}$ dextrose) rather than with L-15 medium. Third, in light of the high concentrations of choline needed for maximal acetylcholine synthesis in these cultures (Patterson and Chun, 1977), the concentration of choline in the perfusate was increased to $30 \mu \mathrm{M}$. Finally, recordings were obtained from neurons that were obviously connected by neuritic bundles within the fields of photomicrographs; neurons that were located at the edges of the field and whose processes appeared to leave the field were not impaled.

A modification (Pickel et al., 1975) of the peroxidaseantiperoxidase method (Sternberger, 1974) was employed for the immunocytochemical localization of TH; details of our method have been described elsewhere (Iacovitti et al., 1980). The specificity of the antiserum to TH that was used in this study has been previously demonstrated in sections obtained directly from animals (Joh et al., 1973; Pickel et al., 1975) and in preparations from tissues grown in culture (Iacovitti et al., 1980).
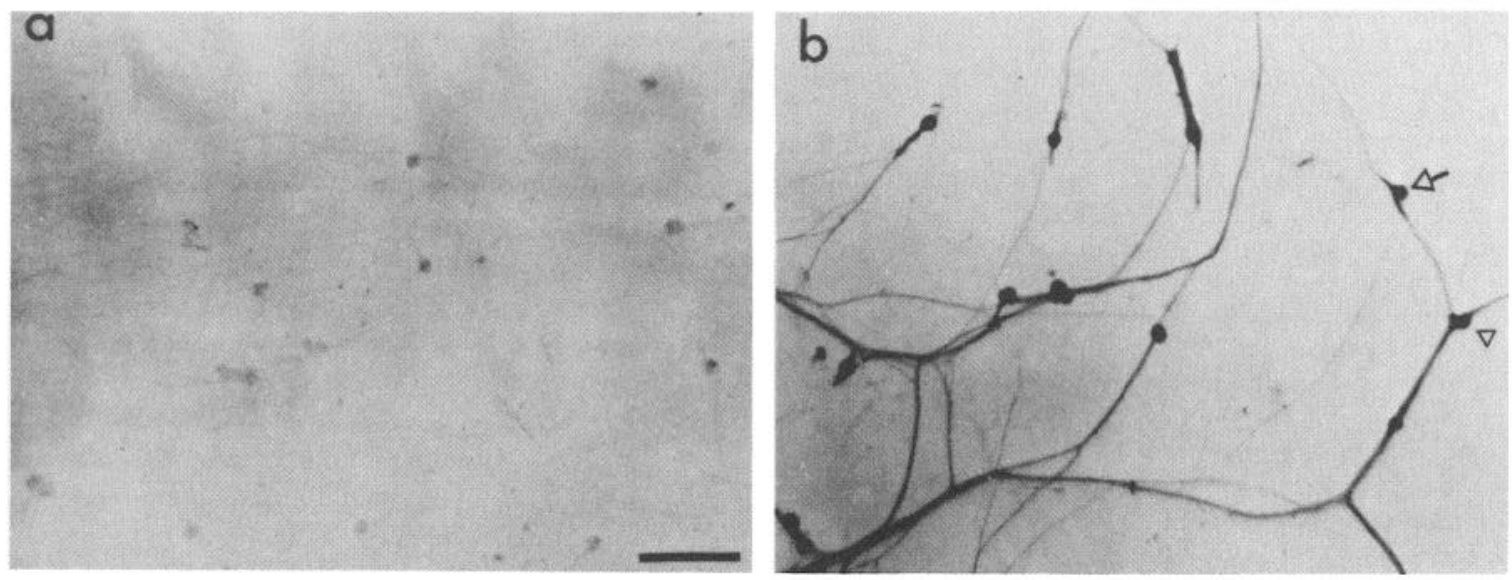

Figure 1. Specificity of the immunocytochemical reaction. $a$, Nonspecific staining in a culture of dissociated SCGN exposed to IgG from pre-immune serum. The bar represents $250 \mu \mathrm{m}$ and applies to both micrographs. $b$, Specific staining of the neurons in a sister culture ( 28 days in vitro) exposed to the antibody to $\mathrm{TH}$. The perikarya of all neurons in this micrograph were judged to have shown a positive reaction for TH. The arrow in $b$ points to a driver neuron; the electrophysiological recordings obtained from this cell are shown in the lower traces in Figure 3. The neuron which was excited by the driver is indicated by the arrowhead. 

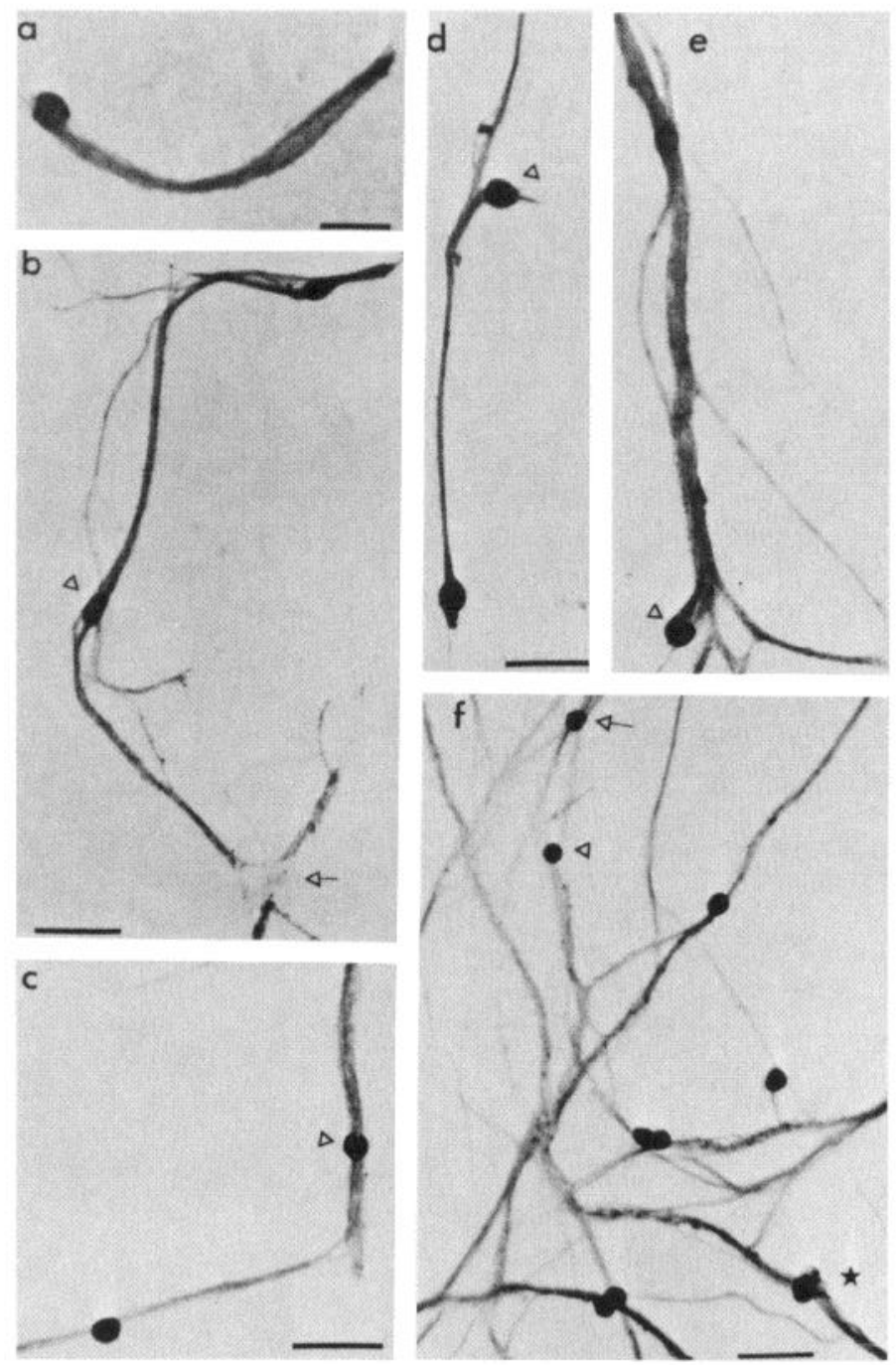

Figure 2. Immunocytochemical localization of $\mathrm{TH}$ within SCGN at various times in vitro. All neurons in these micrographs (except those specifically indicated in $f$ ) were judged to have shown a positive reaction for TH. $a, 16$ days in vitro. Electrophysiological recordings from this cell are shown in Figure $4 a_{1-3} . b, 21$ days in vitro. One cell was lost (arrow) from this field during fixation. Simultaneous recordings from that cell (bottom trace) and from another cell (arrowhead, top trace) in this area are shown in Figure $4, b_{1}$ and $b_{2} . c, 28$ days in vitro. Simultaneous recordings from both cells are shown in Figure 4, $c_{1}$ and $c_{2}$. The top trace was obtained from the cell indicated by the arrowhead. $d, 35$ days in vitro. Simultaneous recordings from both cells in this field are shown in Figure $4, d_{1}$ and $d_{2}$. The top trace was obtained from the cell indicated by the arrowhead. $e, 35$ days in vitro. The arrowhead points to the cell from which the records in Figure $4 e_{1-3}$ were obtained. $f, 49$ days in vitro. There is a cluster of three cells in the lower right corner of this photograph (star). Two of them were scored as showing a negative reaction for TH. The arrow (top trace) and arrowhead (bottom trace) indicate two of the cells from which recordings were obtained (Fig. $4 f$ ). The calibration bar represents $50 \mu \mathrm{m}$ in $a$ and $100 \mu \mathrm{m}$ in $b, c, d$, and $f$. The magnification is the same in $d$ and $e$.

\section{Results}

Tyrosine hydroxylase immunocytochemistry. In each experiment, two controls for nonspecific staining were used. First, clitures of SCGN were processed with IgG from pre-immune serum (Fig. 1a) and second, cultures of tissues other than the SCGN (heart cells, Schwann cells, fibroblasts, and dissociated dorsal root ganglion (DRG) neurons) were processed with the antibody to $\mathrm{TH}$ (data not shown). In agreement with previous findings, only very low levels of nonspecific staining were observed in either control group. As a control for the act of impalement, DRG neurons were impaled with microelectrodes and then exposed to the TH antibody; the level of nonspecific staining was not increased.

In every culture of SCGN that had been exposed to the TH antibody, specific, dark brown deposits were seen within the perikaryon of most cells (Figs. $1 b$ and 2). A region of lighter staining was generally visible over the nucleus in cells that had not been impaled with microelectrodes; typical photomicrographs have been published elsewhere (Iacovitti et al., 1980). In impaled cells, the clear nuclear region was often, but not always, absent. However, the cytoplasmic staining reaction of nearly all impaled SCGN was identical to the predominant staining reaction of the other SCGN on the dish and to the staining reactions of SCGN in sister cultures of a comparable age that had not been subjected to the conditions used for the electrophysiological experiments. Table I shows the incidence of neurons that stained for TH within the areas used for electrophysiological measurements. More than $95 \%$ of the cells that could be relocated showed a positive reaction within the soma. In addition, specific dark brown deposits were noted over all neurite bundles that had been visible in light micrographs before staining. However, as most processes ran in bundles, we cannot comment on the staining properties of individual fibers, nor was it possible to separate axons from dendrites by these methods (Banker and Cowan, 1979; Wakshull et al., 1979a).

Electrophysiology. Intracellular recordings were obtained from 91 neurons from cultures of various ages (Table II, Figs. 3 and 4). Upon stimulation, 77 of these neurons (termed drivers) caused excitatory synaptic potentials of either short $(<5 \mathrm{msec})$ (Fig. 4) or long ( $>5$ msec) latency (Fig. 3). In 14 of 14 experiments with the neurons used for immunocytochemistry, all spontaneous (data not shown) and evoked synaptic potentials (Figs. 3 and 4) were rapidly abolished or reduced by more than $90 \%$ in the presence of 100 or $50 \mu \mathrm{M}$ hexamethonium, respectively. The excitatory potentials were unaffected by atropine $(0.3 \mu \mathrm{M}, n=4)$ or propranolol $(0.3 \mu \mathrm{M}, n=6)$ (Fig. $3 d$ ). No instances of electrical transmission were found and, in agreement with the observations of others (Ko et al., 1976; O'Lague et al., 1978a), no inhibitory synaptic potentials were observed.

The percentage of neurons that functioned as driver cells remained rather constant as a function of time in vitro (Table II). Thus, after 16 days, the earliest time studied, $76 \%$ of the neurons sampled were found to function as drivers; after 7 weeks, $75 \%$ were drivers.

The observations that $95 \%$ of the neurons in cultures of all ages studied have TH within their cytoplasm and that $85 \%$ of the neurons (average of all times in vitro) functioned as drivers, made it highly likely that most driver neurons had $\mathrm{TH}$ within their cytoplasm. That this is the case is shown by the data illustrated in Figures 3 
and 4 and summarized in Table II. Of the 77 drivers studied, 70 remained on the slides and could be relocated after the immunocytochemical procedure. Sixty-nine of these driver neurons showed a positive reaction for $\mathrm{TH}$ (Figs. 1 and 2). Furthermore, the percentage of drivers that stained for TH remained constant from the 16 th to the 49th day in vitro. Interestingly, all neurons that we could not identify as drivers also had $\mathrm{TH}$ within their cytoplasm (Table II).

\section{Discussion}

Iacovitti et al. (1980) found that more than $95 \%$ of the neurons of the embryonic superior cervical ganglion had TH molecules within their cytoplasm when they were maintained in vitro under the present conditions. In agreement with this finding, approximately $95 \%$ of the neurons observed in this study gave a positive reaction for TH; it is, therefore, likely that the restricted areas selected for electrophysiological studies were representative of the total population of neurons in these cultures. The only morphological difference observed in this report was a loss of the lighter staining area over the nuclei in

TABLE I

Number of neurons showing a positive reaction within their perikarya when exposed to the antibody to TH at various times in vitro

On the day before an experiment, micrographs were made of representative areas of cultures. The second column of this table gives the total number of neurons (impaled and unimpaled) observed in the micrographs of the area that were relocated for electrophysiological recordings. The third and fourth columns, respectively, show the number of these neurons that had a positive or negative reaction for TH. A few neurons (column 5) could not be relocated after completion of the immunocytochemical procedure; a typical example of the location of a lost neuron is shown in Figure $2 b$.

\begin{tabular}{ccccc}
\hline Time In Vitro & $\begin{array}{c}\text { No. of } \\
\text { Neurons } \\
\text { Observed }\end{array}$ & $\begin{array}{c}\text { TH } \\
\text { Positive }\end{array}$ & $\begin{array}{c}\text { TH } \\
\text { Negative }\end{array}$ & Lost \\
\hline days & & & & \\
16 & 54 & 43 & 5 & 6 \\
21 & 49 & 42 & 2 & 5 \\
28 & 61 & 52 & 1 & 8 \\
35 & 11 & 11 & 0 & 0 \\
49 & 66 & 59 & 2 & 5 \\
Total of all ages & 241 & 207 & 10 & 24 \\
\hline
\end{tabular}

most of the impaled neurons; this occurred even when recording times were kept to a minimum (less than 10 min). Since impalements were usually obtained by causing the tip of the microelectrode to vibrate, it seems possible that this motion may have damaged the nuclear membrane. Alternatively, it is possible that the impalements caused such injury to the cell that the nuclear changes observed are those characteristic of cell death (Trump and Mergner, 1973).

These results confirm the findings of previous physiological studies (O'Lague et al., 1974, 1978a, b; Ko et al., 1976; Wakshull et al., 1978, 1979b) in showing that nearly all of the excitatory potentials, spontaneous and evoked, observed in cultures of dissociated SCGN are caused by the release of acetylcholine and that these potentials can be specifically inhibited by nicotinic cholinergic antagonists. The short latency responses are most probably
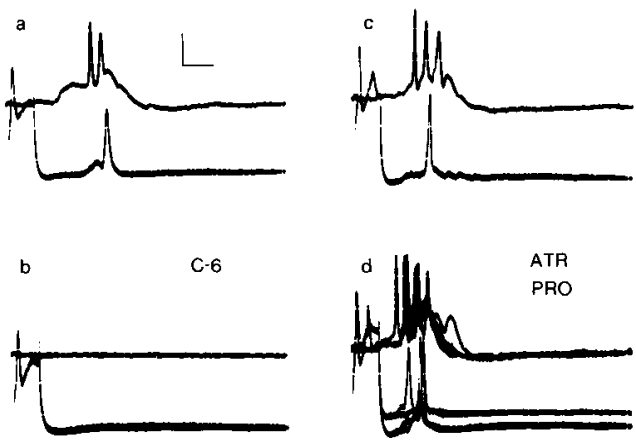

Figure 3. Synaptic interactions blocked by hexamethonium. The records shown in this figure were obtained from the neurons which have been identified in Figure $1 b$. $a$, Stimulation of a driver neuron (lower trace) caused a burst of action potentials and excitatory synaptic potentials (termed complex waves by O'Lague et al., 1978a) to occur after a long latency in both the driver neuron and in another neuron (top trace). $b$, After the addition of hexamethonium $(C-6,50 \mu \mathrm{M})$ to the perfusate, stimulation of the driver failed to evoke complex waves. $c$, Within $3 \mathrm{~min}$ of the removal of hexamethonium, stimulation of the driver again caused complex waves to occur in both neurons. $d$, In the presence of atropine $(A T R, 0.3 \mu \mathrm{M})$ and propranolol $(P R O, 0.3 \mu \mathrm{M})$, the complex waves could still be evoked (three superimposed traces). The vertical calibration bar in a represents $20 \mathrm{mV}$; the horizontal bar represents $50 \mathrm{msec}$. Scale applies to all records.

TABLE II

The detection by immunocytochemical methods of $T H$ within neurons that have been functionally characterized by electrophysiological methods

\begin{tabular}{|c|c|c|c|c|c|c|c|c|c|}
\hline Time In Vitro & $\begin{array}{l}\text { Total No. of } \\
\text { Neurons } \\
\text { Impaled }\end{array}$ & $\begin{array}{l}\text { No. of } \\
\text { Drivers }\end{array}$ & $\begin{array}{l}\text { Driver and } \\
\text { TH Positive }\end{array}$ & $\begin{array}{c}\text { Driver and } \\
\text { TH Nega- } \\
\text { tive }\end{array}$ & $\begin{array}{l}\text { Driver and } \\
\text { Lost }\end{array}$ & $\begin{array}{c}\text { No. of } \\
\text { Non-drivers }\end{array}$ & $\begin{array}{l}\text { Non-driver } \\
\text { and TH } \\
\text { Positive }\end{array}$ & $\begin{array}{l}\text { Non-driver } \\
\text { and TH } \\
\text { Negative }\end{array}$ & $\begin{array}{l}\text { Non-driver } \\
\text { and Lost }\end{array}$ \\
\hline \multicolumn{10}{|l|}{ days } \\
\hline 21 & 26 & 24 & 22 & 0 & 2 & 2 & 2 & 0 & 0 \\
\hline 28 & 18 & 18 & 15 & 0 & 3 & 0 & 0 & 0 & 0 \\
\hline 35 & 6 & 4 & 4 & 0 & 0 & 2 & 2 & 0 & 0 \\
\hline Total of all ages & 91 & 77 & 69 & 1 & 7 & 14 & 12 & 0 & 2 \\
\hline
\end{tabular}



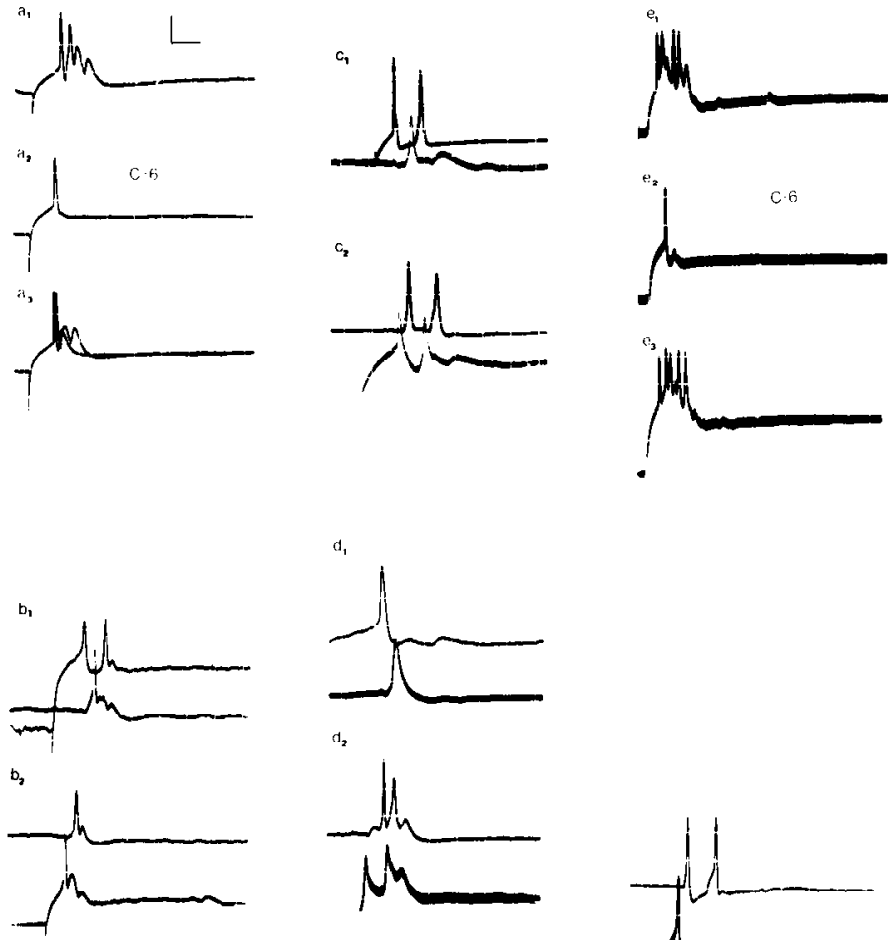

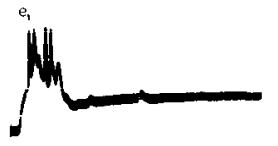

by hexamethonium strictly allows us to conclude only that at least one neuron in the polysynaptic network released acetylcholine. However, in extensive electrophysiological investigations in similar cultures in which thousands of neurons have been impaled (O'Lague et al., 1974, 1978a, b; Ko et al., 1976; Wakshull et al., 1978, $1979 \mathrm{~b}$ ), the only excitatory mode of transmission (other than cholinergic) that has been observed is electrical transmission; none of the neurons in the present study and fewer than $7 \%$ of the cells in previous studies were found to employ such a mode of transmission. It is concluded, therefore, that acetylcholine was probably released by all of the driver neurons that caused long latency responses. ${ }^{3}$

The direct observations obtained from individual neurons with both electrophysiological and immunocytochemical techniques allow three conclusions. First, sympathetic neurons in culture that possess a physiologically demonstrable ability to release acetylcholine can have TH molecules in their perikaryon. Second, under some conditions, this mixed phenotype is not a property of a small minority of the neurons, but rather of nearly all of the neurons. Third, this mixed phenotypic expression can be a stable rather than a transient phenomenon that is manifest by at least the 16th day in vitro and persists for over a month. Since others (Furshpan et al., 1976; Landis, 1976; Reichardt and Patterson, 1977; Wakshull et al., 1978) have observed that physiologically identified cholinergic neurons retain the capacity to transport and release catecholamines, there is now evidence that at least three principal characteristics of adrenergic neurons can be stably expressed by cholinergic neurons in vitro. These results clearly show that these neurons have the potential for dual function in retaining the specific enzymes for nonepinephrine synthesis while they are producing and releasing acetylcholine. At this time, it is unclear whether the presence of mixed transmitter systems within a single neuron is an artifact of the tissue culture environment. It should be noted, however, that Grazanna and Coyle (1978) have presented strong evidence that another adrenergic enzyme, dopamine $\beta$-hydroxylase, can be found within some parasympathetic neurons in the intact animal. Furthermore, Bloom and Edwards (1980) and Lundberg et al. (1980) have indicated that some cholinergic neurons may release a peptide transmitter.

While we have shown that it is possible for parts of two transmitter systems to persist in a neuron, it does not necessarily follow that this dual expression is stable under all conditions in vitro. The apparent discrepancy between our data and that of Reichardt and Patterson (1977) may be attributable to the differences in the nutrient media used by the various investigators. Alternatively, the differences may have been caused by the types of assays that were used. The parameter measured by Reichardt and Patterson is the net result of the

monosynaptic (O'Lague et al., 1978a); we conclude that acetylcholine was released by the driver neuron in these cases. The long latency responses are probably polysynaptic. Since we have not recorded from the first synapse made by the neurons that evoked these responses, we have no direct evidence for the mode of transmission used by these driver neurons. The inhibition
${ }^{3}$ If this extrapolation is not accepted, one is still confronted with a dual function neuron that has TH within its perikaryon and yet excites other neurons by a mechanism that does not involve receptors for catecholamines (Ko et al., 1976; O'Lague et al., 1978b; Wakshull et al., 1979a). 
synthesis, storage, and degradation of catecholamines; changes in any of these processes could yield a negative result. Our immunocytochemical experiments establish only the presence of TH within these neurons. Although this enzyme is active in homogenates (Iacovitti et al., 1980), we do not know yet whether it is active in the neurons and whether it has all of the cofactors needed to bring about catecholamine synthesis. Dopamine $\beta$-hydroxylase activity is, however, increasing at the same time and rate as $\mathrm{TH}$ and choline acetyltransferase in comparable cultures.

The present findings do not indicate the cellular mechanisms that allow a neuron that produces catecholamines in vivo to adopt a cholinergic mode of transmission in vitro. The presence of $\mathrm{TH}$ within these neurons, which have a predominance of round, agranular synaptic vesicles (Johnson et al., 1980), may indicate that these cells have not abandoned the catecholamine enzyme system but rather have put aside or modulated some function characteristic of an adrenergic neuron in vivo. The resolution of this problem may require an understanding of the factor(s) or mechanism(s) that determine how, and possibly which, neurotransmitters are packaged in synaptic vesicles. However, any mechanism, including possible competition for synaptic vesicles in a dual potential neuron, will also have to explain the relationship between the packaging of acetylcholine and the persistence of the biosynthetic enzymes for catecholamines (Iacovitti et al., 1980).

\section{References}

Banker, G. A., and W. M. Cowan (1979) Further observations on hippocampal neurons in dispersed cell culture. J. Comp. Neurol. 187: 469-494.

Bloom, S. R., and A. V. Edwards (1980) Vasoactive intestinal peptide in relation to atropine resistant vasodilation in the submaxillary gland of the cat. J. Physiol. (Lond.) 300: 41-53.

Bocchini, V., and P. Angeletti (1969) The nerve growth factor: Purification as a 30,000-molecular-weight protein. Proc. Natl. Acad. Sci. U. S. A. 64: 787-794.

Burton, H., and R. P. Bunge (1975) A comparison of the uptake and release of $\left[{ }^{3} \mathrm{H}\right]$ norepinephrine in rat autonomic and sensory ganglia in tissue culture. Brain Res. 97: 157-162.

Burton, H., and R. Bunge (1980) Cholinergic and catecholaminergic synapses. In Excitable Cells in Tissue Culture, P. G. Nelson and M. Leberman, eds., Plenum Press, in press.

Furshpan, E. J., P. R. MacLeish, P. H. O'Lague, and D. D. Potter (1976) Chemical transmission between rat sympathetic neurons and cardiac myocytes developing in microcultures: Evidence for cholinergic, adrenergic and dual-function neurons. Proc. Natl. Acad. Sci. U. S. A. 73: 4225-4229.

Grzanna, R., and J. T. Coyle (1978) Dopamine- $\beta$-hydroxylase in rat submandibular ganglion cells which lack norepinephrine. Brain Res. 151: 206-214.

Iacovitti, L., T. H. Joh, D. H. Park, and R. P. Bunge (1980) Dual expression of transmitter synthesis in cultured autonomic neurons. Nature, submitted for publication.

Joh, T. H., C. Geghman, and D. J. Reis (1973) Immunochemical demonstration of increased accumulation of tyrosine hydroxylase protein in sympathetic ganglia and adrenal medulla elicited by reserpine. Proc. Natl. Acad. Sci. U. S. A. 70: 27672771.
Johnson, M. 1., C. D. Ross, M. Meyers, S. L. Spitznagel, and R. P. Bunge (1980) Morphological and biochemical studies on the development of cholinergic properties in cultured sympathetic neurons. I. Correlative changes in choline acetyltransferase and synaptic vesicle cytochemistry. J. Cell Biol. 84: $680-691$

Ko, C. -P., H. Burton, M. I. Johnson, and R. P. Bunge (1976) Synaptic transmission between rat superior cervical ganglion neurons in dissociated cell cultures. Brain Res. 117: 461-485.

Landis, S. (1976) Rat sympathetic neurons and cardiac myocytes developing in microcultures: Correlation of the fine structure of endings with neurotransmitter function in single neurons. Proc. Natl. Acad. Sci. U. S. A. 73: 4220-4224.

Lundberg, J. M., A. Änggárd, J. Fahrenkrug, T. Höfelt, and V. Mutt (1980) Vasoactive intestinal polypeptide in cholinergic neurons of exocrine glands: Functional significance of cuexisting transmitters for vasodilation and secretion. Proc. Natl. Acad. Sci. U. S. A. 77: 1651-1655.

O'Lague, P. H., K. Obata, P. Claude, E. J. Furshpan, and D. D. Potter (1974) Evidence for cholinergic synapses between dissociated rat sympathetic neurons in cell culture. Proc. Natl. Acad. Sci. U. S. A. 71: 3602-3606.

O'Lague, P. H., E. J. Furshpan, and D. D. Potter (1978a) Studies on rat sympathetic neurons developing in cell culture. II. Synaptic mechanisms. Dev. Biol. 67: 404-423.

O'Lague, P. H., D. D. Potter, and E. J. Furshpan (1978b) Studies on rat sympathetic neurons developing in cell culture. III. Cholinergic transmission. Dev. Biol. 67: 424-443.

Patterson, P. H. (1978) Environmental determination of autonomic neurotransmitter functions. Annu. Rev. Neurosci. 1: 1-17.

Patterson, P. H., and L. L. Y. Chun (1977) The induction of acetylcholine synthesis in primary cultures of dissociated rat sympathetic neurons. I. Effects of conditioned medium. Dev. Biol. 56: 263-280.

Patterson, P. H., L. F. Reichardt, and L. L. Y. Chun (1975) Biochemical studies on the development of primary sympathetic neurons in cell cultures. Cold Spring Harbor Symp. Quant. Biol. 40: 389-398.

Pickel, V. M., T. H. Joh, P. M. Field, C. G. Becker, and D. J. Reis (1975) Cellular localization of tyrosine hydroxylase by immunochemistry. J. Histochem. Cytochem. 23: 1-12.

Reichardt, L. F., and P. H. Patterson (1977) Neurotransmitter synthesis and uptake by isolated sympathetic neurons in microcultures. Nature 270: 147-151.

Sternberger, L. A. (1974) Immunochemistry, Prentice-Hall, Englewood Cliffs, NJ.

Trump, B. F., and W. J. Mergner (1973) Cell injury. In The Inflammatory Process, B. W. Zweifach, L. Grant, and R. T. McCluskey, eds., pp. 115-259, Academic Press, New York.

Varon, S., J. Nomura, J. Perez-Polo, and E. Shooter (1972) Isolation and assay of nerve growth factor proteins. In Methods of Neurochemistry, R. Fried, ed., pp. 203-229, Marcel Dekker, Inc., New York.

Wakshull, E., M. I. Johnson, and H. Burton (1978) Persistence of an amine uptake system in cultured rat sympathetic neurons which use acetylcholine as their transmitter. J. Cell Biol. 79: 121-131.

Wakshull, E., M. I. Johnson, and H. Burton (1979a) Postnatal rat sympathetic neurons in culture. I. A comparison with embryonic neurons. J. Neurophysiol. 42: 1410-1425.

Wakshull, E., M. I. Johnson, and H. Burton (1979b) Postnatal rat sympathetic neurons in culture. II. Synaptic transmission by postnatal neurons. J. Neurophysiol. 42: 1426-1436. 